\title{
The many faces of type I interferon in systemic lupus erythematosus
}

\author{
Claudia Mauri and Madhvi Menon \\ Centre for Rheumatology, Division of Medicine, University College London, London, United Kingdom.
}

\begin{abstract}
Systemic lupus erythematosus (SLE) is a multisystem autoimmune disease with a broad spectrum of clinical presentations involving multiple organ systems. An abnormal response to self-antigens is thought to drive the development of SLE; however, the factors that underlie this dysfunction are not clear. In this issue of the $J C I$, Li and colleagues present compelling evidence to show that type I interferons (IFNs) produced by plasmacytoid dendritic cells inhibit the clearance of apoptotic cells (ACs) by marginal zone macrophages. Specifically, type I IFNs increase the translocation of marginal zone $(\mathrm{MZ}) \mathrm{B}$ cells to the follicular region of the spleen, thereby disrupting interactions between these $B$ cells and $M Z$ macrophages (MZMs), which in turn disrupts megakaryoblastic leukemia 1-mediated (MKL1-mediated) mechanosensing and inhibits $A C$ phagocytosis by MZMs. The results of this study provide important insight into factors that inhibit AC clearance and promote the development of SLE.
\end{abstract}

to impaired phagocytosis and defective AC clearance (5). In particular, marginal zone macrophages (MZMs) surrounding the splenic follicles have been reported to play a crucial role in the efficient clearance of ACs and in the induction of tolerance to AC autoantigens (AC-Ags) (6). MZM dysfunction results in a loss of tolerance to self-antigens and a decrease in AC clearance (7).

\section{Type I IFNs in SLE}

Type I IFNs are produced in response to both viral and self-nucleic acids and are primarily secreted by plasmacytoid dendritic cells (pDCs) (8). Whereas many cell types produce IFN $\beta$ and some other subtypes of type I IFNs, IFN $\alpha$ is almost exclusively produced by pDCs $(9,10)$. Chronic activation of nucleic acid-sensing receptors expressed on the surface of pDCs stimulate IFN $\alpha$ overproduction and activation of signals downstream of IFN $\alpha / \beta$ receptors (IFNARs) on target cells. The overproduction of type I IFN has been implicated in the pathogenesis of SLE, as this phenotype has been observed in gene expression studies and serum analysis of patients (11). Moreover, excessive IFN $\alpha$ production contributes to SLE pathogenesis via increased induction of plasma cells, production of autoantibodies, and promotion of $\mathrm{T}$ cell-dependent inflammation (10). While the role of ACs in promoting type I IFN overproduction in SLE is well documented, the effect of excess IFN on the clearance of ACs has remained uninvestigated. In this issue, $\mathrm{Li}$ and colleagues use a combination of genetic mouse models to demonstrate a novel role for type I IFNs in defective MZM-mediated clearance of ACs in SLE (12).

\section{Type I IFNs: dictators of} defective $A C$ clearance

Increased production of type I IFNs by pDCs and decreased clearance of circulating ACs is a common feature of both human patients and mouse models of SLE $(2,11)$. Using two lupus-prone mouse models (BXD2 and B6.Sle1.Sle2.Sle3 [B6.TC] mice), Li et al. 
A

\section{MZ B cell follicle}

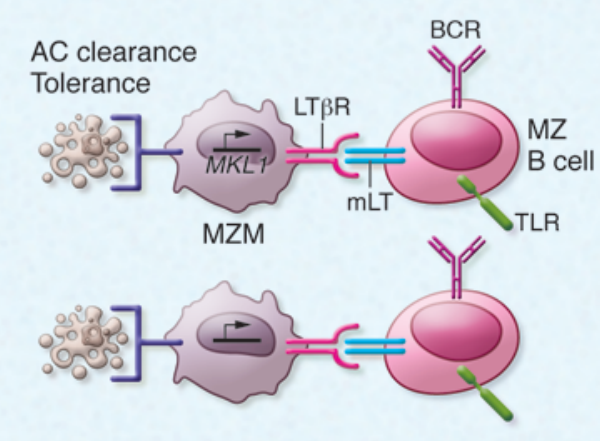

B

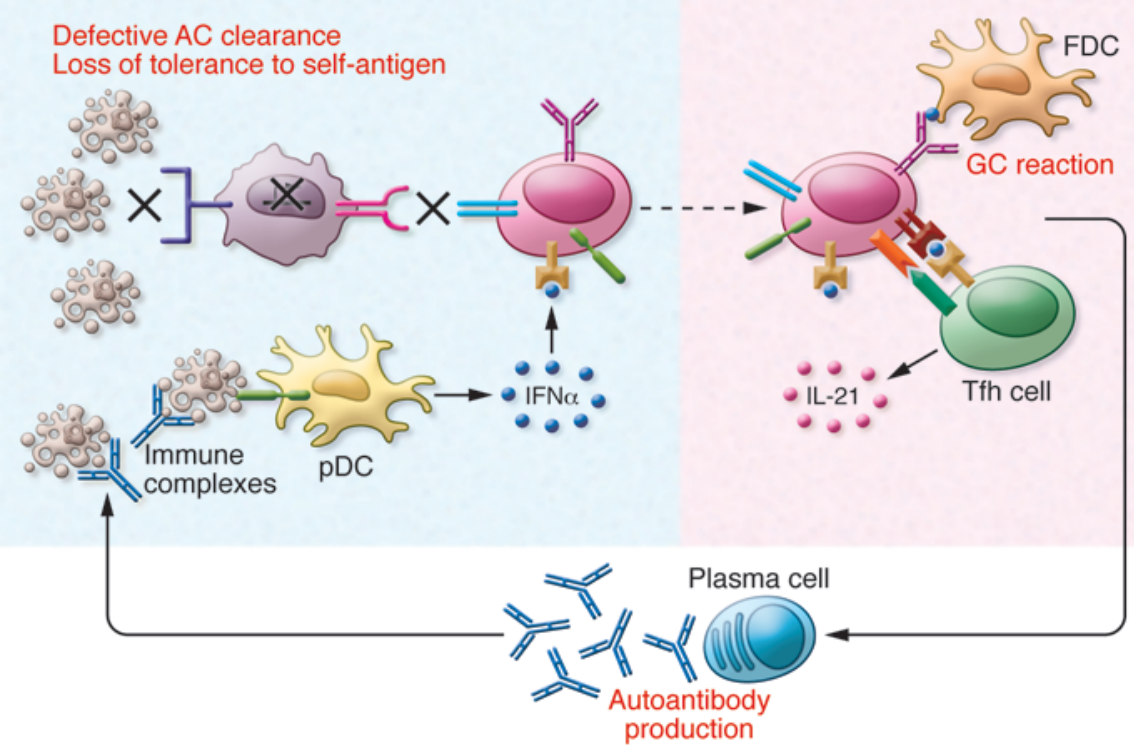

Figure 1. AC clearance in healthy individuals and patients with SLE. (A) mLT-expressing MZ B cells interact with LT $\beta R$-expressing MZMs in the perifollicular region of the spleen, which promotes MKL1 expression by MZMs and maintains their numbers and phagocytic function. (B) In lupus spleens, $\mathrm{pDCs}$ accumulate at the perifollicular region and produce IFN $\alpha$, which induces the migration of $\mathrm{mLT}^{+}$ $M Z B$ cells to the follicle. This results in the loss of MZM numbers and function, leading to defective $A C$ clearance and loss of tolerance to self-antigens. Furthermore, MZ B cells in the follicle interact with FDCs and T follicular helper ( $T f h$ ) cells and stimulate germinal center reactions that result in autoantibody production. The antinuclear autoantibodies form immune complexes to further stimulate pDCs to produce type I IFNs. Amplification of these defective interactions contributes to and perhaps initiates the pathogenesis of SLE.

found that an increase in pDCs is paralleled by a decrease in MZMs in the perifollicular region of the spleen (12). Remarkably, analysis of the distribution of MZMs and pDCs in the spleens of patients with SLE showed a similar pattern. Specifically, compared with healthy controls, patients with SLE exhibited a decrease in MZMs and an accumulation of pDCs in the spleen. Interestingly, IFNAR deficiency in BXD2 mice restored MZM numbers and prevented pDC aggregation in the spleen, suggesting that IFNAR signaling may promote lupus by dissipating phagocytic MZMs in the spleen (12). However, at this stage, the possibility that reduced MZM numbers in the spleens of patients with SLE are a consequence of different therapies cannot be excluded.

To further investigate the dynamics of the interaction between MZ B cells, MZMs, and type I IFN during the development of lupus, BXD2-Rag ${ }^{-1}$ mice were reconstituted with bone marrow from either $\mathrm{GFP}^{+}$ BXD2-Ifnar ${ }^{+/+}$or GFP'- BXD2-Ifnar ${ }^{--}$mice.
Analysis by confocal microscopy revealed that, whereas $\operatorname{IgM}^{\text {hi }} C D 1 d^{\text {hi }} M Z$ B cells derived from the bone marrow of IFNAR ${ }^{+}$ mice had translocated to the follicle, those from IFNAR-deficient mice were retained in the MZ. These results demonstrate that the translocation of MZ B cells to the follicle is dependent on type I IFN signaling. There was no difference in the percentages of MZMs in the spleens of mice that received IFNAR-deficient bone marrow compared with the percentages in recipients of IFNAR-sufficient bone marrow, indicating that type I IFN does not directly affect MZM numbers or function. Additionally, Li et al. depleted MZ B cells to assess whether MZ B cell translocation affected MZM numbers and function. Indeed, administration of antibodies against the NOTCH2 ligand delta-like 1 (DLL1), which is crucial for MZ B cell development (13), to SLE mice resulted in depletion of MZ B cells as well as a marked reduction in the number of MZMs (12). As NOTCH2 is not expressed by MZMs, these results imply that MZ B cells are required for the maintenance of homeostatic levels of splenic MZMs in lupus-prone mice. These results raised the question of whether and how type I IFN regulates the numerical distribution of MZ B cells, which directly or indirectly affects the frequency of MZMs during lupus progression. Previous studies have implicated membrane lymphotoxin (mLT) expression by B cells in the maintenance of MZMs (14), as mLT is highly expressed on MZ and MZ-precursor $\mathrm{B}$ cells. Li and colleagues took advantage of a combination of IFNAR-deficient, lupusprone mice and conditional knockout mice lacking $\mathrm{mLT}$ expression exclusively on B cells to demonstrate that the translocation of $\mathrm{mLT}^{+} \mathrm{B}$ cells out of the MZ into the follicle is driven by type I IFNs, resulting in a decrease in MZMs (12). These results highlight the importance of mLT-expressing MZ $B$ cells in the maintenance of MZM numbers and phagocytic function.

\section{$\mathrm{mLT} / \mathrm{LT} \beta \mathrm{R}$ signaling, loss of MZMs, and the development of lupus}

$\mathrm{Li}$ and colleagues also detailed a molecular mechanism for the defective mechanosensing of signals by MZMs and the reduced MZM numbers in lupus. Specifically, the authors demonstrated that the interaction between lymphotoxin $\beta$ recep- 
tor (LT $\beta \mathrm{R}$ ) expressed on MZMs and $\mathrm{mLT}$ on the surface of MZ B cells is pivotal for the maintenance of MZMs. Loss of LT $\beta R$ signaling on macrophages resulted in alterations in the number and function of MZMs and led to the development of lupus-like disease (12). These results are in agreement with those of previous studies showing that disruption of the interaction between MZMs and $\mathrm{mLT}^{+} \mathrm{B}$ cells occurs in lupus-prone BXD2 and B6.TC mice (15, 16). Li et al. demonstrated that LT $\beta R$ signaling maintains expression of a mechanosensing transcriptional coactivator, megakaryoblastic leukemia 1 (MKL1) (17, 18 ), and that the activation of this signaling cascade is important for MZM homeostasis in the MZ as well as for clearance of ACs by MZMs (12). Moreover, MKL1-deficent mice displayed a decrease in MZMs and developed a spontaneous lupus-like disease, elegantly confirming the importance of the mLT/LT $\beta R$ pathway and associated MKL1 expression in preventing lupus.

Type I IFN has been shown to promote the translocation of $\mathrm{mLT}^{+} \mathrm{B}$ cells from the MZ to the follicles, where they can stimulate a spontaneous germinal center response by interacting with follicular dendritic cells (FDCs) (19). This result, coupled with previous reports that follicular translocation of AC-Ag-bearing MZ B cells results in the induction of autoreactive $\mathrm{T}$ cells $(20,21)$, highlights a shift in $\mathrm{mLT}^{+} \mathrm{MZ}$ $B$ cells from a tolerogenic response in the $\mathrm{MZ}$ to an immunogenic response in the follicles that is driven by type I IFNs. Taken together, Li and colleagues highlight an important role of type I IFNs in the initiation of lupus, one that promotes defective AC clearance, loss of tolerance to self-antigens, and autoimmune germinal center reactions (Figure 1 ).

\section{Conclusions}

The study by Li et al. (12) attributes additional pathogenic functions to type I IFNs in SLE. Type I IFNs not only disrupt the interactions between MZMs and MZ B cells, but also induce the translocation of MZ B cells into the follicles, leading to a consequential misplacement of MZMs. The similar loss of MZMs in the spleens of both mice with lupus as well as patients with SLE suggests that this disruption may be an important disease-promoting mechanism in humans. More work will need to be done to address whether neutralization of type I IFN signaling can restore the defects and promote AC clearance. While such an approach may partly restore MZM function, it is unlikely to completely ameliorate disease, as the interaction of MZ B cells with FDCs would continue to promote autoimmune germinal center responses. One possibility to overcome this limitation would be the administration of combination therapy that includes rituximab (anti-CD20), followed by sifalimumab (anti-IFN $\alpha$ ). While rituximab depletes pathogenic B cells (22), sifalimumab would block IFN $\alpha$-mediated signaling (23). Importantly, repopulated $\mathrm{mLT}^{+}$ MZ B cells would be able to interact with MZMs and thereby promote AC clearance and stop the progression of lupus. The use of reagents targeting the SRF/MKL1 axis in MZMs, as suggested by Li et al. (12), may also be beneficial. In conclusion, this study highlights a novel pathogenic role of type I IFNs in defective AC clearance by MZMs in systemic autoimmunity.

\section{Acknowledgments}

We would like to thank P.A. Blair for his comments on the manuscript. M. Menon is funded by a Wellcome Trust project grant (090406/Z/09/Z, to C. Mauri).

Address correspondence to: Claudia Mauri, Centre for Rheumatology, Division of Medicine, University College London, 5 University Street, London WC1E 6JF, United Kingdom. Phone: 44.0.2031082155; E-mail:c.mauri@ucl.ac.uk.

1. Rahman A, Isenberg DA. Systemic lupus erythematosus. N Engl JMed. 2008;358(9):929-939.

2. Shao WH, Cohen PL. Disturbances of apoptotic cell clearance in systemic lupus erythematosus. Arthritis Res Ther. 2011;13(1):202.

3. Casciola-Rosen LA, Anhalt G, Rosen A. Autoantigens targeted in systemic lupus erythematosus are clustered in two populations of surface structures on apoptotic keratinocytes. J Exp Med. 1994;179(4):1317-1330.

4. Isenberg DA, Manson JJ, Ehrenstein MR, Rahman A. Fifty years of anti-ds DNA antibodies: are we approaching journey's end? Rheumatology (Oxford). 2007;46(7):1052-1056.

5. Li Y, Lee PY, Reeves WH. Monocyte and macrophage abnormalities in systemic lupus erythematosus. Arch Immunol Ther Exp (Warsz). 2010;58(5):355-364.

6. McGaha TL, Chen Y, Ravishankar B, van Rooijen N, Karlsson MC. Marginal zone macrophages suppress innate and adaptive immunity to apoptotic cells in the spleen. Blood. 2011;117(20):5403-5412.

7. Li H, et al. Cutting Edge: defective follicular exclusion of apoptotic antigens due to marginal zone macrophage defects in autoimmune BXD2 mice. J Immunol. 2013;190(9):4465-4469.

8. Fitzgerald-Bocarsly P, Dai J, Singh S. Plasmacytoid dendritic cells and type I IFN: 50 years of convergent history. Cytokine Growth Factor Rev. 2008;19(1):3-19.

9. Fitzgerald-Bocarsly P, Feng D. The role of type I interferon production by dendritic cells in host defense. Biochimie. 2007;89(6-7):843-855.

10. Niewold TB, Clark DN, Salloum R, Poole BD. Interferon $\alpha$ in systemic lupus erythematosus. J Biomed Biotechnol. 2010;2010:948364.

11. Banchereau J, Pascual V. Type I interferon in systemic lupus erythematosus and other autoimmune diseases. Immunity. 2006;25(3):383-392.

12. $\mathrm{Li} \mathrm{H}$, et al. Interferon-induced mechanosensing defects impede apoptotic cell clearance in lupus. J Clin Invest. 2015;125(7):2877-2890.

13. Moriyama Y, et al. Delta-like 1 is essential for the maintenance of marginal zone B cells in normal mice but not in autoimmune mice. Int Immunol. 2008;20(6):763-773.

14. Tumanov A, et al. Distinct role of surface lymphotoxin expressed by B cells in the organization of secondary lymphoid tissues. Immunity. 2002;17(3):239-250.

15. Wang JH, et al. Extension of the germinal center stage of B cell development promotes autoantibodies in BXD2 mice. Arthritis Rheum. 2013;65(10):2703-2712.

16. Zhou Z, Niu H, Zheng YY, Morel L. Autoreactive marginal zone $\mathrm{B}$ cells enter the follicles and interact with $\mathrm{CD} 4{ }^{+} \mathrm{T}$ cells in lupus-prone mice. BMC Immunol. 2011;12:7.

17. Miralles F, Posern G, Zaromytidou AI, Treisman R. Actin dynamics control SRF activity by regulation of its coactivator MAL. Cell. 2003;113(3):329-342.

18. Vartiainen MK, Guettler S, Larijani B, Treisman R. Nuclear actin regulates dynamic subcellular localization and activity of the SRF cofactor MAL. Science. 2007;316(5832):1749-1752.

19. Myers RC, King RG, Carter RH, Justement LB. Lymphotoxin $\alpha 1 \beta 2$ expression on B cells is required for follicular dendritic cell activation during the germinal center response. Eur J Immunol. 2013;43(2):348-359.

20. Mountz JD, Wang JH, Xie S, Hsu HC. Cytokine regulation of $\mathrm{B}$-cell migratory behavior favors formation of germinal centers in autoimmune disease. Discov Med. 2011;11(56):76-85.

21. Wang JH, et al. Type I interferon-dependent CD86(high) marginal zone precursor B cells are potent T cell costimulators in mice. Arthritis Rheum. 2011;63(4):1054-1064.

22. Thatayatikom A, White AJ. Rituximab: a promising therapy in systemic lupus erythematosus. Autoimmun Rev. 2006;5(1):18-24.

23. Higgs $\mathrm{BW}$, et al. A phase $1 \mathrm{~b}$ clinical trial evaluating sifalimumab, an anti-IFN- $\alpha$ monoclonal antibody, shows target neutralisation of a type I IFN signature in blood of dermatomyositis and polymyositis patients. Ann Rheum Dis. 2014;73(1):256-262. 